Blockchains and Cryptocurrencies

Trends in building fungible blockchains for data and value exchange

Surekha Thota and Gopal Krishna Shyam

DOI: $\underline{10.1504 / / J B C .2021 .117811}$

\section{Article History:}

Received:

Last revised:

Accepted:

Published online:
International Journal of Blockchains and Cryptocurrencies

ISSN online: 2516-6433 - ISSN print: 2516-6425

https://www.inderscience.com/ijbc 


\title{
Trends in building fungible blockchains for data and value exchange
}

\section{Surekha Thota* and Gopal Krishna Shyam}

\author{
School of Computer Science and Engineering, \\ REVA University, \\ Bengaluru, India \\ Email: surekha.thota@reva.edu.in \\ Email: gopalshyambabu@gmail.com \\ *Corresponding author
}

\begin{abstract}
Industries applaud blockchain technology because of its nature of removing intermediaries involved in assets and value exchange. The first and second-generation blockchain operates in silos and restricts inter-blockchain communication. This restriction is a hindrance to implement blockchain for the real-world asset and value exchange. So, the third generation blockchains focus on issues like scalability and interoperability. We believe that a solution for interoperability can solve the complexities involved in providing blockchain-as-a-service (BaaS). Hence, we reviewed various projects that aim to build fungible blockchains. Each project uses its protocol stack for achieving interoperability. In this research, we conducted a thorough literature review and identified the strengths and weaknesses of various blockchain interoperable projects. We have classified these projects based on the interoperability scheme and finality consensus. This survey helps the researchers and industries to identify the research gaps and choose the best suitable project for their research and business needs.
\end{abstract}

Keywords: interoperability; cross-chain communication; pegged sidechains; BTC Relay; Cosmos; Polkadot; Wanchain; AION; BlockNet; Block Collider; finality consensus; blockchain.

Reference to this paper should be made as follows: Thota, S. and Shyam, G.K. (2021) 'Trends in building fungible blockchains for data and value exchange', Int. J. Blockchains and Cryptocurrencies, Vol. 2, No. 1, pp.83-101.

Biographical notes: Surekha Thota received her BTech in Electrical and Electronics Engineering and MTech in Computer Networks and Information Security from the Jawaharlal Nehru Technological University, Hyderabad. She is currently working as an Assistant Professor in the School of Computer Science and Engineering, REVA University, Bengaluru, India. Her research interests include blockchain technology and cloud computing. She has 11 years of IT industry experience, and for past three years she is in teaching and research.

Gopal Krishna Shyam is currently working as a Professor in the School of Computer Science and Engineering, REVA University, Bengaluru, India. He received his $\mathrm{BE}$, MTech and $\mathrm{PhD}$ from the Visvesvaraya Technological University, Belagavi. He has handled several subjects for UG/PG students like algorithms, computer networks, web programming, information security, computer concepts and $\mathrm{C}$ programming. His research interest includes cloud 
computing, grid computing and high-performance computing. He is a member of the IEEE and ACM and is actively involved in motivating students/faculties to join professional societies. He has an experience of around 13 years in teaching and research.

\section{Introduction}

Blockchain is a distributed ledger that has the potential to operate in a trustless environment (Nakamoto, 2008). The capabilities of achieving immutability and transparency in a decentralised ecosystem, eliminating intermediaries, and providing faster settlement world-wide make blockchain best suitable for enterprises. Recent years witnessed that blockchain is the latest and greatest breakthrough for enterprise solutions.

Hundreds of silo blockchains are available in the market to work for a specific purpose. The decentralised applications on a blockchain can only access digital assets on that particular blockchain. This limitation is a hindrance when multiple blockchains are involved in a transaction. This situation occurs especially when multiple stakeholders are involved or cross-border transactions takes place. Interoperability is the ability to exchange information seamlessly between different blockchain networks. Interoperability accelerates the blockchain adaptation to real-world applications (Ducrée, 2020). Hence, there is a need to build fungible blockchains that provides seamless transfer of information.

Based on the business need, interoperability among various blockchains is required to transfer:

1 the financial assets

2 information

3 both information and financial assets.

Different blockchain platforms use different consensus algorithms (Lone and Mir, 2019; Karthika and Jaganathan, 2019) like:

1 Bitcoin - proof of work (PoW) (Nakamoto, 2008)

2 Ethereum - proof-of-stake (PoS) (Wood, 2014)

3 Hyperledger Sawtooth - proof of elapsed time (Olson et al., 2018) and so on.

This heterogeneity among various platforms creates additional complexity to design interoperable blockchains. Not only there exist differences in consensus algorithms, but they differ in their native cryptocurrencies [Bitcoin-Bitcoin (Nakamoto, 2008), Ethereum-Ether (Wood, 2014), Ripple-XRP (Schwartz et al., 2014)]. Furthermore, in Bitcoin, there is a maximum limit on the number of BTC's that can be generated (21 million), and even there lies a restriction that only 12.5 BTC's are created and credited to the miner as a coin base transaction on block creation (Nakamoto, 2008). As the coins are limited, we should neither destroy them on the sender blockchain nor create equivalent coins on the receiver's blockchain. An interoperable solution should not duplicate, create or destroy the digital assets. This opens up research on digital asset management and its transactions. 
Enterprises are exploring blockchain solutions for their business problems, due to which hundreds of unconnected blockchains have been born. To promote blockchain-as-a-service (BaaS), there is a definite need to:

1 connect different blockchain systems

2 connecting blockchain with the traditional legacy system.

In brief, the prerequisite to implement $\mathrm{BaaS}$ is to have interoperability between heterogenous blockchain platforms. Enterprises are facing a lot of technical and business challenges while providing solutions for blockchain interoperability (Kim et al., 2020). This brings more research scope for interoperability between blockchains.

In this paper, we review various blockchain and the technical innovations in building the fungible blockchain. Section 2 discusses various projects that support interoperability. Section 3 compares and summarises these projects. We attempt to categorise these projects based on the interoperability scheme and the finality guaranteed by them. In Section 4, we conclude the paper and provide a future scope.

\section{Technical innovations}

To review the technical innovations in building interoperable blockchains, we have assimilated information from various sources like academic journals, conference papers, white papers, online articles/blogs and recorded videos. In this section, we discuss some of the blockchain interoperability projects and compare our study with the existing literature.

\subsection{Pegged sidechains}

Sidechains are considered the first interoperable solution that allows the transfer of digital assets between multiple blockchains. In one-way peg sidechains (Buterin, 2016; Back et al., 2014), the participant has to destroy his BTC by sending it to an unspendable address on the blockchain. In turn, he receives XCP tokens (a native currency of counterparty). 'Proof-of-burn' creates XCP tokens. The main drawback is that one-way peg is an irreversible transaction, due to which the XCP tokens cannot convert back to BTC.

To address the above-stated problem, sidechains extended to a two-way peg (Back et al., 2014), which allows the bidirectional transfer of assets between the parent and sidechains. Instead of sending the BTC to an unspendable address, a two-way peg locks asset and supplies the tokens used for transacting in the side chains. These tokens are converted back to BTC by submitting the cryptographic simplified payment verification (SPV) proof as proof for locking the assets.

Another flavour of sidechain implementation is a federated sidechain (Back et al., 2014; Dilley et al., 2016). A federation is a group of servers that intermediate between the main chain and side chains. Federations decide whether to lock or release the transactions. A federated model replaces the dynamic membership multiparty signature (DMMS) by miners with a fixed multi-signature set by federations. Although this approach reduces latency issues, it leaves a risk that federated pegs may become 
centralised and trustless, until strong federations are maintained. This opens up research scope to move towards decentralisation and build strong federations.

\subsection{BTC Relay}

BTC Relay (Buterin, 2016; Chow, 2016) tries to achieve interoperability between Ethereum and Bitcoin. Using BTC Relay, the dapps on the Ethereum platform can accept Bitcoin payment. Ethereum contract stores the Bitcoin block header. When an Ethereum dapp receives a Bitcoin payment, it validates the transactions using SPV. It is a technique that verifies and validates the transactions using block headers without the need for a full blockchain. Relayers submit the block headers to the BTC Relay. Relayers are rewarded for verifying transactions.

BTC Relay replaces the third party with a trust-minimised smart contract. BTC Relay provides one-way interoperability between Ethereum and Bitcoin, meaning that Bitcoin cannot read the Ethereum chain as there is no Ethereum relay contract on Bitcoin. This opens up research scope to build a two-way relay communication.

\subsection{Ripple}

Financial institutions struggle to have fast, transparent, and seamless cross-border transactions. Ripple Labs (Schwartz et al., 2014) addresses this issue using inter ledger protocol (ILP). ILP (Thomas and Schwartz, 2015) is an open-source protocol for universal cross-payments. It promises faster transactions by connecting the ledgers of various banks and eliminates the need for centralised authorities. When the sender and receiver are not on the same payment network, ILP uses multiple connectors to pass the payment till it reaches the receiver as shown in Figure 1. ILP uses a cryptographic escrow mechanism with a two-phase commit that transfers the funds among various distributed ledgers. In a two-phase commit, the first step is to lock the funds and define a set of conditions for the execution and cancellation of transactions. And the second step is to process the transactions when the set conditions are satisfied.

Figure 1 Ripple interoperability (see online version for colours)

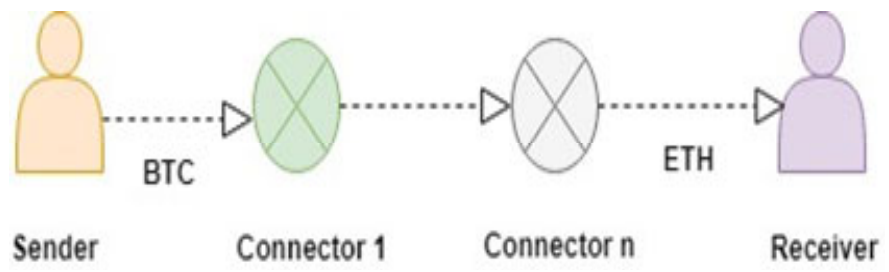

The advantage of Ripple is its high processing speed of transactions and low transactional fee. The main limitation is that it does not cater to non-payment applications. XRP coins are pre-mined (Johnson et al., 2018) and so no incentives are given for the nodes. Ripple company partly controls the XRP tokens, which may compromise the sole benefits of decentralisation. The research scope here is to extend ripple to non-payment applications as well. 


\subsection{Cosmos}

Cosmos (Kwon and Buchman, 2018) is an interconnection of independent blockchains built on top of Tendermint. Its application layer interacts with a low-level layer (Tendermint Core) using a socket protocol called the application blockchain interface $(\mathrm{ABCI})$. Tendermint Core consists of two sub-layers:

1 the consensus layer (PoS BFT)

2 the networking layer (P2P gossip protocol).

Cosmos addresses the challenges of both scalability and interoperability. But our focus is to discuss the concepts that support interoperability.

Cosmos architecture's design has two classes, i.e., hub and zones, as shown in Figure 2. Cosmos hub is a centralised hub that connects all the blockchains (zones). Zones are heterogeneous blockchains that wish to exchange data and value with other blockchains. Zone connects to the hub using inter-blockchain communication (IBC) protocol.

Figure 2 Cosmos architecture (see online version for colours)

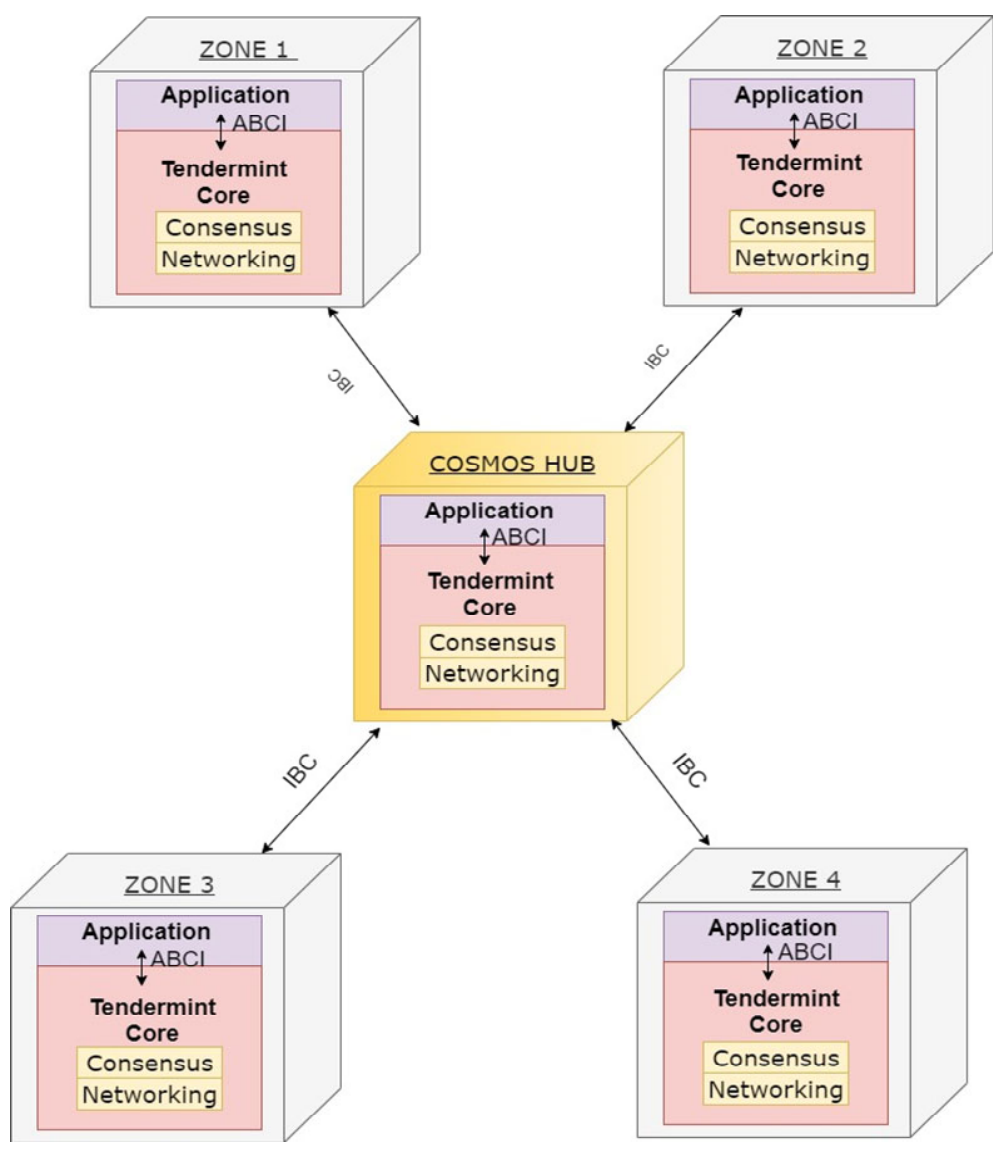

Note: Each zone is a blockchain. 
Zones connect to centralised hubs, which in turn connect to every zone connected to that hub. Instead of connecting to each zone individually, Cosmos with a single connection (from the zone to the hub) provides access to all zones connected to that hub. It reduces the number of connections required for the interconnection of blockchains. Hub prevents double-spending, which means, when a zone receives a token from the hub, it has to trust only the sending zone of this token.

In the crypto world, finality is the affirmation that once a block is added to the blockchain, it will not be reverted. In probabilistic-finality (provided by Bitcoin's PoW), the possibility of transaction reversion decreases as the length of the blockchain gets deeper. However, in fast-finality or absolute finality (provided by Tendermint), the transaction is immediately considered final, as it is added to blockchain (Gauba, 2018).

While providing interoperability, the coupling varies between fast-finality and probabilistic-finality consensus. The blockchain that uses fast-finality can connect to Cosmos hub using IBC protocol, however, the blockchains that use probabilistic-finality (PoW) connect using peg-zones (Cosmos, https://cosmos.network/intro).

In Tendermint, every validator has to communicate with each other to either approve or reject a single block, thus creating finality at the per-block level. The algorithm is fast and has been stress-tested in a live environment with 200 validators and six-second block time during game of stakes (Julian, 2019). If the number of validators increases, communication overhead is high between the validators, and therefore the average block creation time is increased drastically. This opens up research scope on how to reduce average block creation time with increase in number of validators.

\subsection{Polkadot}

Polkadot (Wood, 2016) is an interoperable blockchain infrastructure for Web3. Polkadot is written in Rust language and developed by Parity. Polkadot (2018) architecture decouples the consensus architecture from the application. Polkadot uses a hybrid consensus mechanism with Aurand and Tendermint practical Byzantine fault tolerance (PBFT). Aurand provides fast and intermediate finality, whereas Tendermint PBFT provides absolute finality. After a series of blocks added with Aurand, the PBFT checkpoint guarantees the finality (Polkadot, 2018).

Tendermint guarantees the finality of every ' $n$ ' block, while the ' $n-1$ ' in-between blocks are processed quickly without the need for 2/3's consensus (Polkadot, 2018). This hybrid design depicted in Figure 3 allows much faster consensus, and yet sometimes, the proposed blocks can be reverted if it is an invalid block.

Figure 3 Polkadot hybrid consensus (see online version for colours)

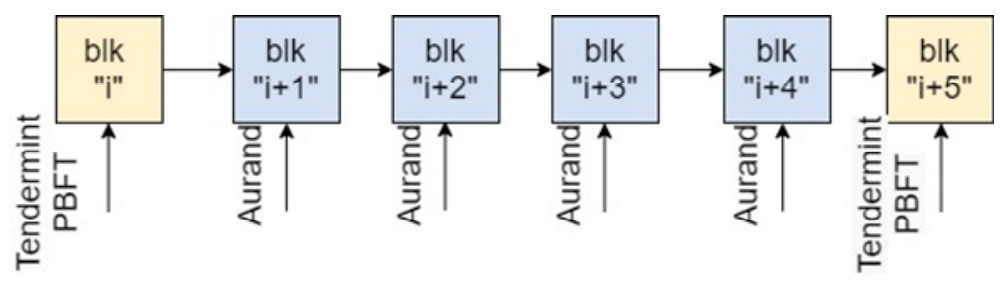

Note: In this example $\mathrm{n}=4$, four blocks will be added through Aurand consensus, while $\mathrm{n}^{\text {th }}$ block uses Tendermint PBFT. 
Polkadot network is a PoS blockchain, and its native tokens are 'Dots'. The network's central hub is the 'relay chain' and the blockchains connecting to it are 'parachains'. Polkadot allows shared pool security between parachains and the relay chain. New parachains are attached to the Polkodot network by accumulating a large number of Dots. Parachains can be removed from the network by detaching these dots.

The polkadot network has four main stakeholders namely validators, nominators, collators and fishermen. Collators have to maintain the full nodes. They can store and validate the new parachain blocks. They execute zero-knowledge proof and provide it to validators, who propose parachain block to relay chain. Fishermen get rewarded by submitting the proofs of illegal blocks created by validators or collators. A nominator is a stakeholding party that contributes to the security bond of a validator.

Figure 4 Polkadot stakeholders (see online version for colours)

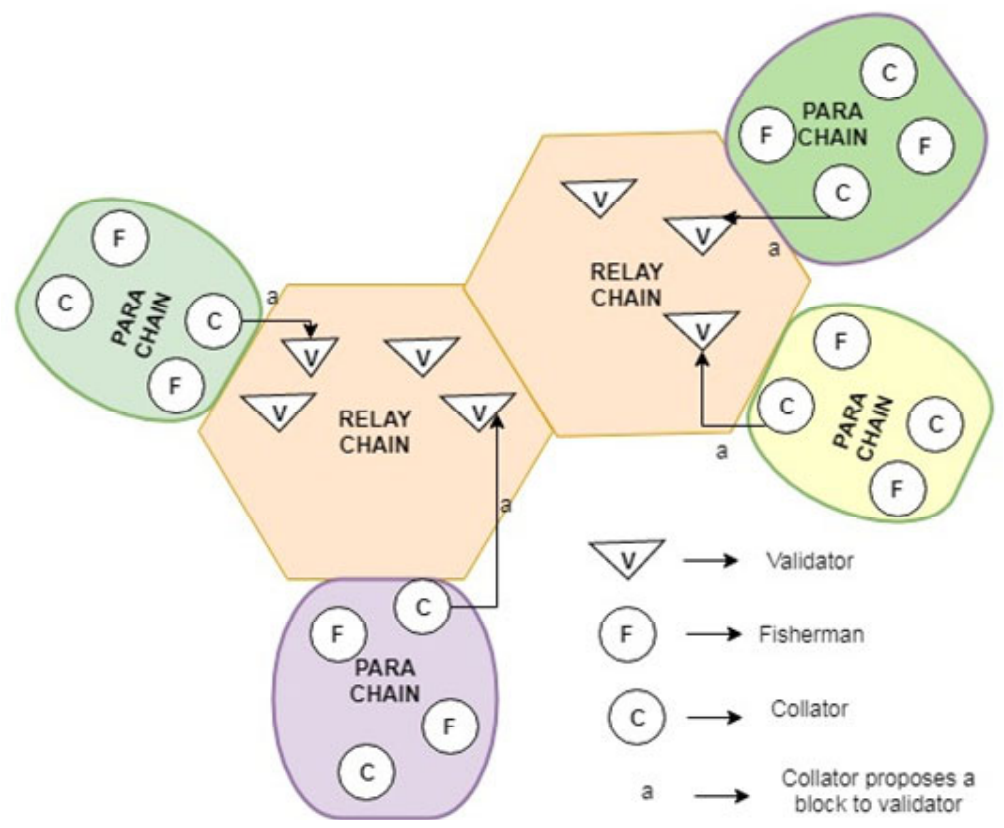

Table 1 Similarities between Polkadot and Cosmos

\begin{tabular}{lcc}
\hline Parameter & Polkadot & Cosmos \\
\hline Consensus & $\begin{array}{c}\text { Hybrid consensus mechanism } \\
\text { with Aurand and Tendermint }\end{array}$ & Tendermint \\
Nodes & Validators, nominators, & Validators, delegators \\
& collators and fishermen & \\
Central connector & Relay Chain & Cosmos hub \\
Blockchains & Parachains & Cosmos zones \\
Connectivity to blockchains & Bridges & Peg zones \\
Native tokens & Dots & Atoms \\
\hline
\end{tabular}


Polkadot and Cosmos are very similar in their architecture. Table 1 shows these similarities. Polkadot requires 100 validators to secure a relay-chain and five parachains, whereas the Cosmos network requires 100 hub validators and 500 zone validators (Dave, 2018). Thus, the Polkadot interoperability is much easier than the Cosmos. Since Polkadot's power lies in Dot ownership, it is less decentralised than the Cosmos.

\subsection{Wanchain}

Building a fully interoperable blockchain network will be the core of the digital economy. Wanchain aims to build a financial market that interoperates with different digital assets. Bitcoin and Ethereum are the widely used cryptocurrencies. Wanchain provided interoperability with Ethereum in Wanchain 2.0 and with Bitcoin in Wanchain 3.0 release.

Wanchain (https://wanchain.org/files/Wanchain-Whitepaper-EN-version.pdf) is an Ethereum-based ledger that uses many original Ethereum functions. To accommodate interoperability, Wanchain uses cross-chain communication protocol and smart contract token privacy protection.

Wanchain supports ordinary transactions using PoS consensus and cross-chain transactions using secure multi-party computation (SMPC) and threshold key exchange (TKE). SMPC technique (Maizels, 2018) fragments one secret key and distributes it over several nodes so that no one knows anything about the secret key. To retrieve the secret key, a minimum number of users must pool their data together. Suppose the secrete key is built using ' $n$ ' fragments, and a minimum of ' $t$ ' fragments are sufficient to reconstruct your secret key. Sharing of these minimum thresholds is TKE (Reecer, 2018).

Following are the sequence of steps to be followed when a cross-chain transaction is being initiated from ETH to WAN:

1 The currency on initiating blockchain (ETH) should not be destroyed, rather it has to be made unavailable by locking the funds indefinitely in a hashed time-locked contract (HTLC).

2 The equivalent value should be made available on the destination chain (Wanchain, https://wanchain.org/files/Wanchain-Whitepaper-EN-version.pdf) as a mapping token (WETH).

3 The locked Ethereum funds (Step 1) is released when the WETH holder transfers WETH to a HTLC account

Wanchain's storeman nodes are responsible for the cross-chain transactions (locking and releasing funds). They employ the SMPC method with a threshold protected secret key to process cross-chain transactions. They are responsible for the generation of locked account's public and private keys. SMPC and TKE prevent a malicious storeman from carrying out a double-spend attack. A minimum of key fragments is required for key reconstruction. If storemen lose a key share or go offline, it is still possible to broadcast a transaction (Maizels, 2018).

Privacy protection in Wanchain is supported by using ring signatures and one-time addresses (OTAs). The anonymity of the signer is provided by ring signatures and the anonymity of the receiver is provided by OTAs. Wanchain eliminates single point failure and supports multiple cryptocurrencies. In addition to WAN coins, Wanchain wallet extends its support to Ethereum, Bitcoin and ERC-20 tokens. 
Wanchain's weakness is that it relies on PoS, which is theoretically subject to a threat of collusion attack of validator nodes. This opens up research scope in building a threat free validator nodes.

\section{$2.7 \quad A I O N$}

The interoperability solution proposed by AION is a hub and spoke model. The hub in AION is the main blockchain that connects all participating blockchains via bridges (Spoke and NE Team, 2017). Although AION uses a centralised hub, it does not lead to a single point of failure as the hub itself is a blockchain. AION uses a hybrid PoW/PoS consensus (Wu et al., 2019). In PoW, the user with more hash power mines the block, and in PoS, the user with high stakes likely has a chance to propose a new block. Hybrid PoW/PoS consensus interleaves blocks produced by both algorithms into one chain.

The long-term goal of AION is to have a new neural network verification algorithm called proof-of-intelligence (Culwick and Metcalf, 2018). A proof-of-intelligence is to enable deep neural network capabilities on top of AION, so that it eventually runs artificially intelligent applications on top of the blockchain.

\subsection{BlockNet}

Most of the above discussed interoperable projects are centralised entities in blockchain, which compromise the whole idea of decentralised peer-to-peer blockchain. BlockNet aims to preserve the decentralised approach of the blockchain. Nodes that support inter-chain communication are present both on their network edge and on the blockchain network edges that they communicate. It runs in a self-sovereign manner.

BlockNet (Weisman, 2018) uses three principles for inter-chain infrastructure:

1 nodes distributed to the edges of its network

2 nodes distributed to the edges of the participating network

3 self-sovereign delivery and consumption of services.

Three main components for inter-chain communication in BlockNet:

- Blockchain router (XName): An ecosystem of inter-chain services requires a mechanism to route messages to the correct blockchain via the inter-chain address system. It provides an ability to look up and query the services provided by a particular blockchain, defines a routing standard (DNS), and uses a proof scheme to provide truthfulness.

- Inter-chain network overlay (XBridge): XBridge is a serverless distributed hash table (DHT)-based peer-to-peer network and the ability to interconnect nodes of different blockchain networks, building an inter-chain network overlay. It looks up and locates the peers residing on any blockchain network, then sends a broadcast message.

- Peer-2-peer data transport (XChat): It provides an ability to send and receive the order for data and value exchange on the blockchain. XChat provides end-to-end encryption for a peer-to-peer messaging module that supports both unicast and multicast messages. Steps for service lookup are: 
1 Discover peers on XBridge.

2 Identify and get the service's chain Id and service list using XName.

3 Query to find a peer on the designated chain Id using XBridge.

4 Xchat retrieves the list of the services provided by the peer.

5 Request a service in a self-sovereign manner.

BlockNet uses fully decentralised exchange. It decentralises all four components deposits, order broadcasting, order matching and exchanging of coins. Hence, user has full control on their currency.

\subsection{Block Collider}

Block Collider (Curran, 2019) is on multichain. Block Collider's initial launch provides interoperability with five products Bitcoin, Ethereum, NEO, Lisk, and Waves. Block Collider is an interchain blockchain protocol that uses a modified version of PoW known as proof of distance (PoD) (Block Collider Team, 2018; Curran, 2019).

Block Collider weaves several blockchains into a single blockchain (the block collider chain). This single resultant intermediary blockchain represents the perfect state of all blockchains. It forms by mining the header hash of other blockchains using edit distance (ED). Miners compete to find a string whose hash value in a normalisation process must be less than a minimum distance threshold.

Let us consider three participating blockchains that subsequently mine to create a single intermediary blockchain. In the process of mining, we take the header hash of each blockchain (say h1, h2, h3) and set the threshold to ' $\mathrm{t}$ '. Using proof of ED, the miner should find a hash string ' $\mathrm{B}$ ' that is less than ' $\mathrm{t}$ ' of all the blockchains (Explore Proof of Edit Distance, http://s3.amazonaws.com/blockcollider/lab/poed.html). This is represented mathematically in equation 1.

Equation (1): Proof of ED

$$
\begin{aligned}
& \mathrm{ED}(\mathrm{H},(\mathrm{h} 1), \mathrm{H}(\mathrm{B}))<\mathrm{t} \& \& \mathrm{ED}(\mathrm{H}(\mathrm{h} 2), \mathrm{H}(\mathrm{B}))<\mathrm{t} \& \& \mathrm{ED}(\mathrm{H}(\mathrm{h} 3), \mathrm{H}(\mathrm{B}))<\mathrm{t} \\
& ==\text { true }
\end{aligned}
$$

The same is depicted in Table 2 and Table 3 and calculated using Deng et al. (2018). Consider three participating blockchains Ethereum, Bitcoin and NEO. Table 2 depicts their block header hash, and the threshold set to 0.2888 .

\begin{tabular}{|c|c|}
\hline Blockchain & Hash of block header \\
\hline Ethereum & 0x73413ff99013f6007b72e299aee87da63311602ae4dfb1466b254b7c8A9b8e1bd \\
\hline Bitcoin & 0000000000000000006abfe31e59af9f81b3fc84a1a25a8fdc095e429dc6dffa \\
\hline NEO & 0b58e3e1980eb79c293ee1d047997c5a7092da8d49813c2407e90f85e0ba1f9e \\
\hline
\end{tabular}

Table 2 Inputs for proof of edited distance

On mining, the PoD algorithm calculates the edited distance of each of the participating blockchain and generates a single block hash as shown below 25ce8309cdfdc63df1d0bea789b2ee6c2781b249795c3c40d178c54d61f001b5.

While calculating the edited distance, ensures that it is less than 0.2888 (threshold). The values of the edited distance shown in Table 3. 
Table 3 Proof of ED less than threshold

\begin{tabular}{lc}
\hline Blockchain & Edit distance \\
\hline Ethereum & 0.27884615384615385 \\
Bitcoin & 0.27884615384615385 \\
NEO & 0.28525641025641024 \\
\hline
\end{tabular}

Block Collider blocks do not have a fixed number of transactions. Instead, each block has a distance balance. The sum of the block's transaction distances must stay below the number of emblems the block miner owns. Thus, it is PoD.

Table 4 Comparison with existing literature

\begin{tabular}{|c|c|c|c|}
\hline Paper details & Contribution & $\begin{array}{c}\text { Classification based } \\
\text { on interoperability } \\
\text { scheme }\end{array}$ & $\begin{array}{l}\text { Classification } \\
\text { based on } \\
\text { finality }\end{array}$ \\
\hline $\begin{array}{l}\text { Monika and } \\
\text { Bhatia (2020) }\end{array}$ & $\begin{array}{l}\text { Interoperability is classified into } \\
\text { - Notary scheme solutions } \\
\text { - Sidechain/relay solutions } \\
\text { - Smart contract solutions } \\
\text { - Bridging solutions } \\
\text { - Blockchain router solutions }\end{array}$ & $\mathrm{Y}$ & $\mathrm{N}$ \\
\hline $\begin{array}{l}\text { Belchior et al. } \\
(2020)\end{array}$ & $\begin{array}{l}\text { The major category of } \\
\text { interoperability solutions of review is } \\
\text { - Cryptocurrency-directed } \\
\text { interoperability approaches } \\
\text { - Blockchain engines } \\
\text { - Blockchain connector }\end{array}$ & $\mathrm{Y}$ & $\mathrm{N}$ \\
\hline $\begin{array}{l}\text { Johnson et al. } \\
\text { (2019) }\end{array}$ & $\begin{array}{l}\text { Discussed various products that } \\
\text { contribute to interoperability }\end{array}$ & $\mathrm{N}$ & $\mathrm{N}$ \\
\hline $\begin{array}{l}\text { Monrat et al. } \\
\text { (2019) }\end{array}$ & $\begin{array}{l}\text { Comparison different categories of } \\
\text { blockchain and different consensus } \\
\text { algorithm }\end{array}$ & $\mathrm{N}$ & $\mathrm{N}$ \\
\hline $\begin{array}{l}\text { Hegnauer } \\
(2019)\end{array}$ & $\begin{array}{l}\text { Discussed interoperability techniques } \\
\text { - Notary schemes } \\
\text { - sidechains } \\
\text { - Hash-locking } \\
\text { - Other projects }\end{array}$ & $\mathrm{Y}$ & $\mathrm{N}$ \\
\hline Current paper & $\begin{array}{l}\text { Discussed } 9 \text { products and classified } \\
\text { them based on } \\
\text { - Interoperability scheme } \\
\text { - Finality } \\
\text { Discussed about centralised and } \\
\text { decentralised exchanges }\end{array}$ & $\mathrm{Y}$ & Y \\
\hline
\end{tabular}

Note: $\mathrm{Y}$ - explicit classification and $\mathrm{N}$ - no explicit classification. 
Block Collider uses PoD and eliminates the need for validators for inter-chain communication. In Bitcoin's PoW, all the blocks have fixed-size. Block Collider uses a concept by leveraging its macro asset called emblems that enable miners with more emblems to create a larger sized block, thus facilitating a variably sized block.

Unlike Polkadot or Cosmos, the Block Collider does not require any modification to the bridged chains. The average block interval of Ethereum is roughly 30 seconds, and Bitcoin is 10 mins. As the Block Collider depends on bridged chains, the block interval is dynamic.

Industries appreciate blockchain because of its immutable and transparent nature. Hence, while achieving interoperability, it is essential to retain the immutability aspect of the blockchain. Hence, we made a thorough literature review of relevant papers and compared with our study in Table 4 . We analysed that some of the discussed literature had dealt with classifying the products based on various interoperability schemes. But there is no discussion on how these blockchains are achieving immutability. Immutability in the blockchain is identified through finality. Hence in this paper, we classified the blockchain products both on interoperability scheme and on finality which is discussed in the below section.

\section{Comparative study}

In this paper, we have studied various projects that support interoperability. Table 5 and Table 6 show a comparative study of discussed designs and frameworks concerning interoperability. Further, we have taken a step to classify these projects based on interoperability scheme and consensus finality. We have also discussed that which type of exchange is less vulnerable.

\subsection{Interoperability scheme}

While achieving interoperability between two blockchains $\mathrm{A}$ and $\mathrm{B}$, if an event occurs on $\mathrm{B}$, then the confirmation is be received on ' $\mathrm{A}$ ' and vice versa. This confirmation can be achieved using any one of the following strategies (Back et al., 2014; McConlogue, 2017):

- Notary mechanism: It uses a trusted entity or group to confirm that the claim about blockchain B is correct.

- Relay chain: Instead of depending on the trusted entity, they validate the event on other blockchains. It is called light client verification.

- Hash locking: It uses hash time locking contracts for facilitating atomic swaps between the blockchains A and B. It eliminates the need for intermediaries (Deng et al., 2018).

- Multichain weaving: Rather than having blocks from just a single blockchain, it assembles the blocks from multiple participating blockchains and weaves them into a single chain. 
Table 5 Summary of interoperability projects (part-1) covered under our research

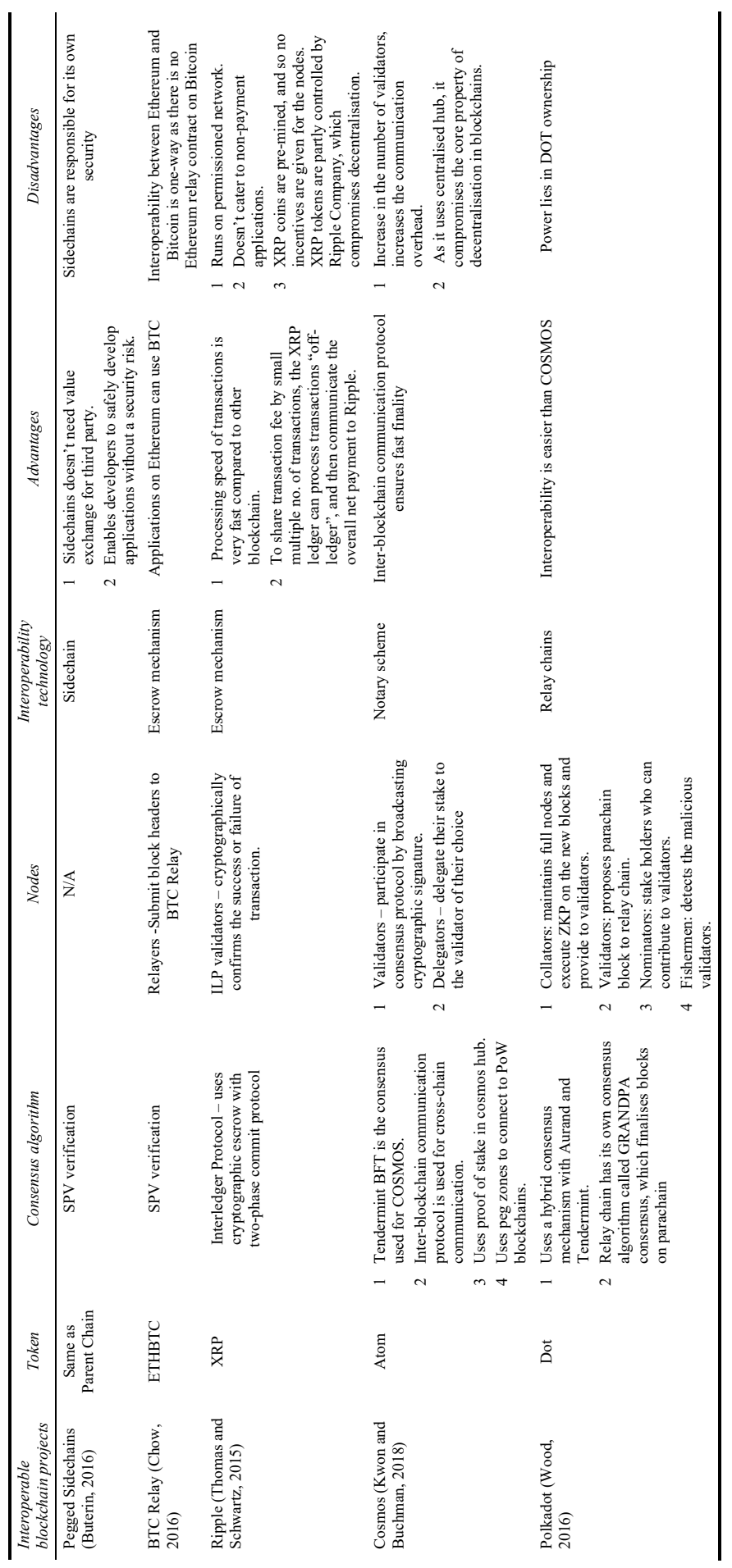


Table 6 Summary of interoperability projects (part-2) covered under our research

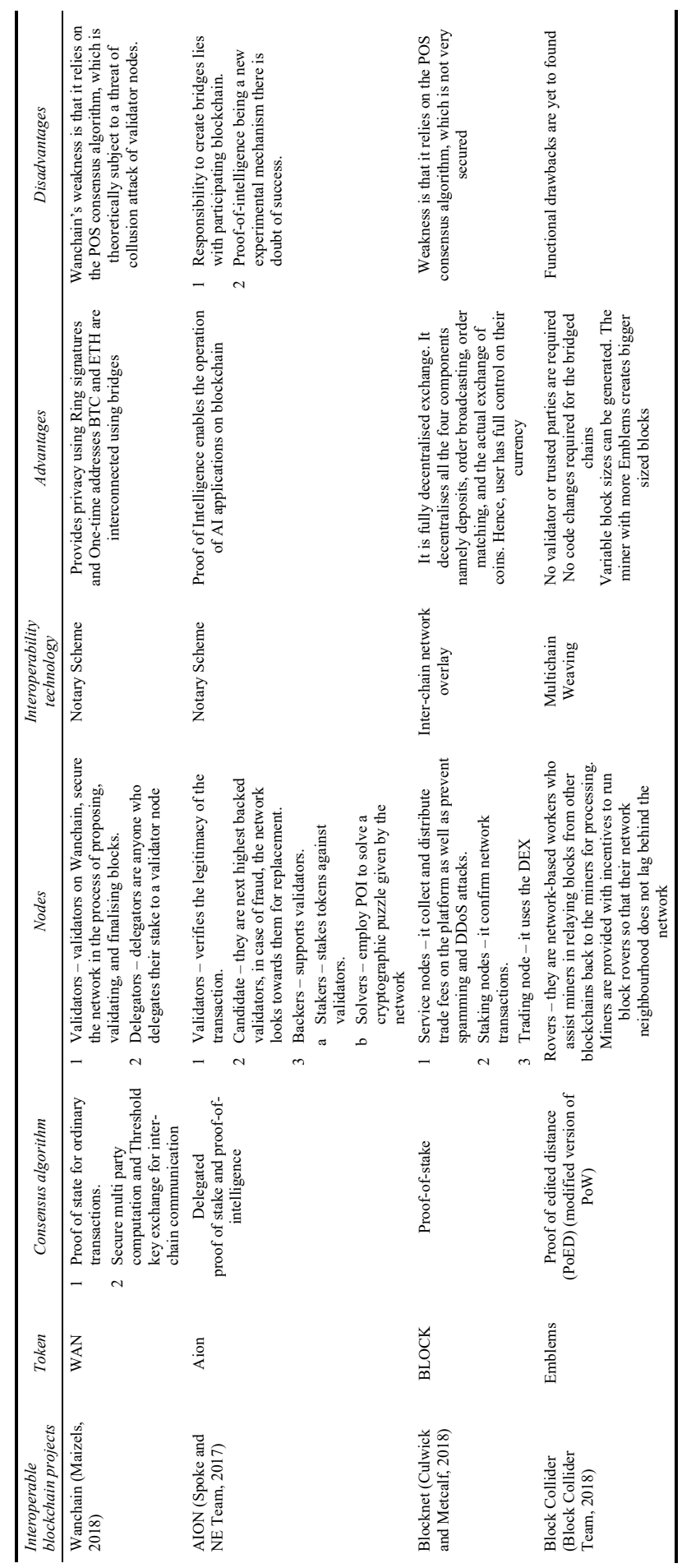


In a notary scheme, whenever the user accesses a dapp or protocol on a different chain, possible scenarios may arise:

- user may have to pay for accessing data on the centralised blockchain

- user may have to pay using hub $\mathrm{n} / \mathrm{w}$ token

- $\quad$ user may have to pay to verify whether the data is truthful or final.

All the above cases lead to vendor locking that compromises the decentralising aspect of blockchain. We have classified projects based on interoperability scheme in Figure 5.

\subsection{Based on the finality of consensus algorithm}

Blockchain had predominantly gained its importance because of its immutability feature. As the depth of the blockchain increases, the older blocks are difficult to tamper with. 'Finality' is the affirmation about the irreversibility of blocks. Following are the various finality notions (Gauba, 2018):

- Probabilistic finality: In this finality, there is a possibility that the transactions may be modified when $51 \%$ of the stakeholders comprise. In probabilistic finality, there is a possibility that a block may be reverted even after being mined. Although it cannot guarantee immediate finality, finality is assured as the block becomes older and older.

- Absolute finality: As soon as the block is added to the blockchain, the transactions are immediately considered final. Tendermint is an example of providing fast finality.

- Economic finality: This notion applies to PoS. If a staker double signs on two blocks, his/her entire stake is slashed out. Hence, it is a loss for the staker and thus referred to as economic finality.

Figure 5 Classification based on interoperability scheme (see online version for colours)

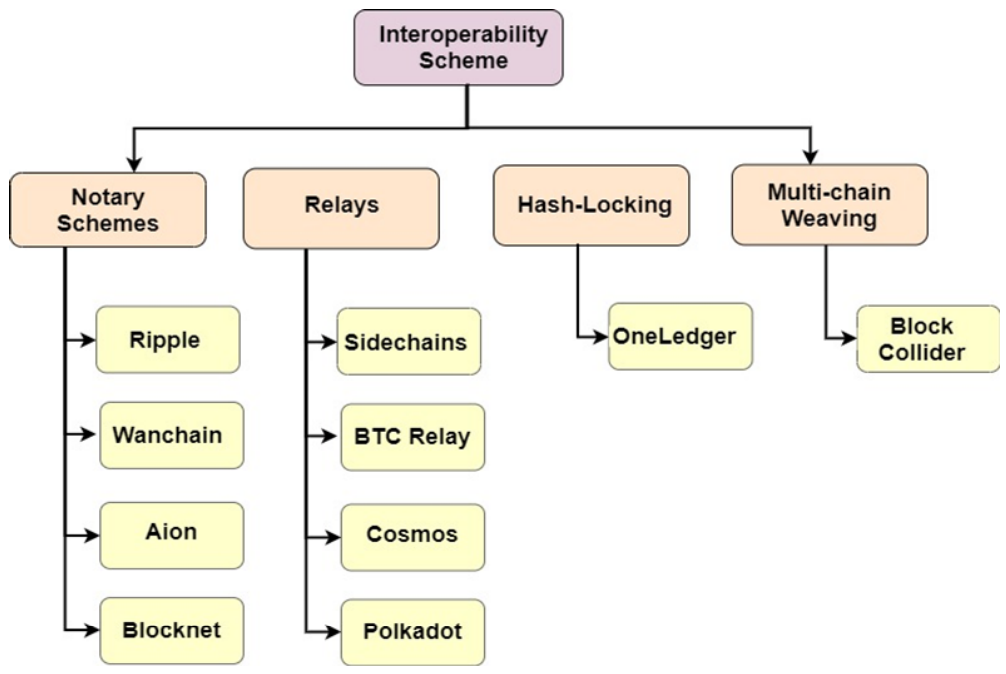


Figure 6 Classification based on finality (see online version for colours)

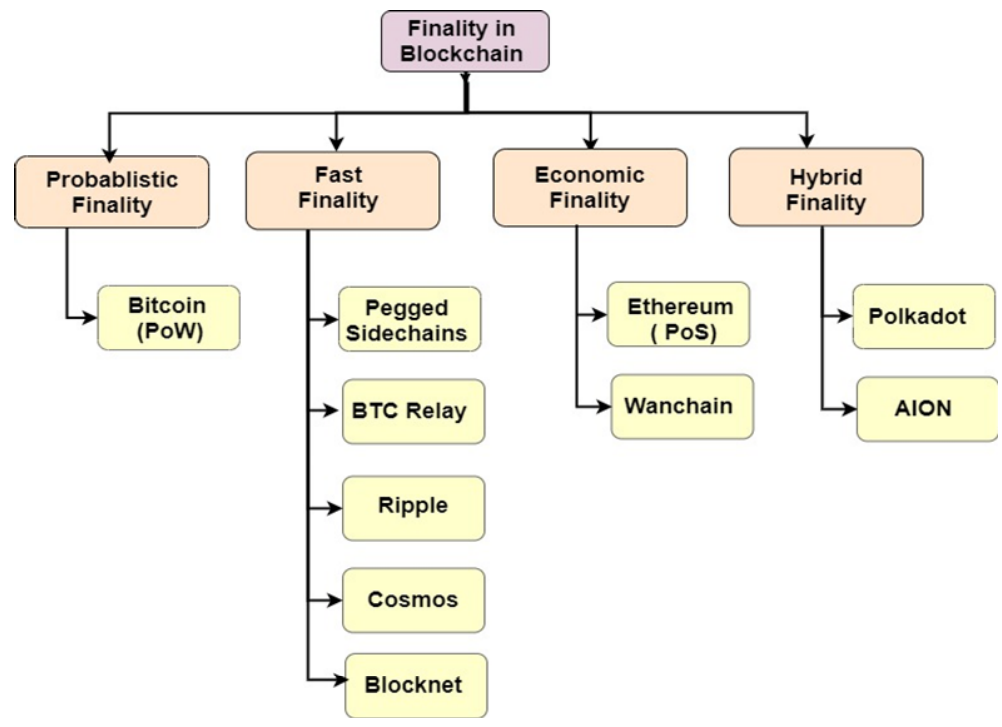

We have classified the above-discussed projects based on type of finality in Figure 6 . However, Block Collider is an alternative to fast finality. Any malicious miners attempting to reorganise the blockchain would need to reverse the entire Block Collider chain along with the hash power difficulty of the targeted bridged chain.

As per our analysis, most of the projects guarantee fast finality whereas some are trying to achieve hybrid consensus. In this context, Block Collider stands unique, as it has to reverse the entire Block Collider chain along with the hash power difficulty, in case if a malicious user tries to tamper with the data.

\subsection{Type of exchanges}

Exchanges are the platforms used to buy and sell cryptocurrencies. Although blockchains are decentralised, they can use either centralised or decentralised exchanges. Bitcoin blockchain uses centralised exchange that involves a middle man or third party to carry transactions. For example, MT.GOX is a centralised bitcoin exchange launched in 2010. By 2013, it was holding 70\% of BTC transactions worldwide. However, in February 2014, it had filed for bankruptcy protection claiming approximately 850,000 Bitcoins belonging to customers and the company were missing and stolen.

To overcome such vulnerabilities, present in centralised exchanges many projects are moving towards decentralised exchanges. Decentralised exchanges eliminate middlemen and carry out transactions using smart contracts and atomic swaps. Decentralised exchanges provide anonymity and ensure an equal degree of trustlessness. Most of the projects under our study are slowly moving towards decentralised exchanges. 


\section{Conclusions}

Researchers are trying to design an efficient mechanism for cross-chain communication for quite some time. It is hard to conclude which of these projects will be more efficient, as it highly depends on adoption, network effectiveness, and delivery of a working project. Hence, this survey presented the strengths and weaknesses of nine different blockchain projects that support interoperability. Further, we have classified them based on the interoperability scheme and consensus finality. Our paper helps the researchers to analyse the current shortcomings to be further researched. It also helps the industries to identify the right blockchain for their business need.

Our survey found that the Block Collider's multi-chain weaving is the most encouraging interoperable solution based on interoperability scheme and finality. We also have observed, that most of the projects are focusing on decentralised exchanges, as the centralised exchanges are prone to vulnerabilities.

Our study brings some of the potential research directions such as:

1 today's decentralised exchanges cannot compete with the transactional speed of centralised exchanges

2 decentralised exchanges do not have a clear jurisdiction

3 lack of standardisation and regulatory guidelines that ensure interoperability between multiple blockchain implementations.

\section{References}

Back, A., Corallo, M., Dashjr, L., Friedenbach, M., Maxwell, G., Miller, A., Poelstra, A., Timón, J. and Wuille, P. (2014) Enabling blockchain Innovations with Pegged Sidechains, Paper, Vol. 72 [online] http://www.opensciencereview.com/papers/123/enablingblockchaininnovations-with-pegged-sidechains (accessed 26 April 2020).

Belchior, R., Vasconcelos, A., Guerreiro, S. and Correia, M. (2020) A Survey on Blockchain Interoperability: Past, Present, and Future Trends, arXiv preprint arXiv: 2005.14282.

Block Collider Team (2018) Block Collider White Paper [online] https://s3.amazonaws.com/ blockcollider/blockcollider_wp.pdf (accessed 28 March 2020).

Buterin, V. (2016) Chain Interoperability, R3 Research Paper [online] https://allquantor.at/ blockchainbib/pdf/vitalik2016chain.pdf (accessed 25 April 2020).

Chow, J. (2016) BTC Relay [online] https://buildmedia.readthedocs.org/media/pdf/btc-relay/latest/ btc-relay.pdf (accessed 30 April 2020).

Culwick, A. and Metcalf, D. (2018) The Blocknet Design Specification [online] https://arlynculwick.com/blocknet-design-specification (accessed 20 March 2020).

Curran, B. (2019) What is Block Collider's Proof of Distance (PoD) Consensus? [online] https://blockonomi.com/block-collider-proof-of-distance-consensus/ (accessed 29 March 2020).

Dave, K. (2018) Blockchain Interoperability: Cosmos vs. Polkadot [online] https://medium. com/@davekaj/blockchain-interoperability-cosmos-vs-polkadot-48097d54d2e2 (accessed 8 February 2020).

Deng, L., Chen, H., Zeng, J. and Zhang, L.J., (2018) 'Research on cross-chain technology based on sidechain and hash-locking', International Conference on Edge Computing, Springer, pp.144-151. 
Dilley, J., Poelstra, A., Wilkins, J., Piekarska, M., Gorlick, B. and Friedenbach, M. (2016) Strong Federations: An Interoperable Blockchain Solution to Centralized Third-party Risks, arXiv preprint arXiv: 1612.05491.

Ducrée, J. (2020) 'Research - a blockchain of knowledge?', Blockchain: Research and Applications, Vol. 1, Nos. 1-2, p.100005 [online] https://doi.org/10.1016/j.bcra.2020.100005.

Explore Proof of Edit Distance [online] http://s3.amazonaws.com/blockcollider/lab/poed.html (accessed 30 March 2020).

Gauba, A. (2018) Finality in Blockchain Consensus [online] https://medium.com/mechanismlabs/finality-in-blockchain-consensus-d1f83c120a9a (accessed 21 February 2020).

Hegnauer, T. (2019) Design and Development of a Blockchain Interoperability API, University of Zurich.

Johnson, N., Prabhav, J. and Anant, J. (2018) Advantages and Disadvantages of Ripple. How Ripple Works [online] https://www.commonlounge.com/discussion/ 1b8f0c74da7042b2a52b2ba84019b35e (accessed 17 April 2020).

Johnson, S., Robinson, P. and Brainard, J. (2019) Sidechains and Interoperability, arXiv preprint arXiv: 1903.04077.

Julian, K. (2019) 5 Differences Between Cosmos \& Polkadot [online] https://medium. com/@juliankoh/5-differences-between-cosmos-polkadot-67f09535594b (accessed 12 March 2020).

Karthika, V. and Jaganathan, S. (2019) 'A quick synopsis of blockchain technology', International Journal of Blockchains and Cryptocurrencies, Vol. 1, No. 1, pp.54-66.

Kim, H.M., Turesson, H., Laskowski, M. and Bahreini, A.F. (2020) 'Permissionless and permissioned, technology-focused and business needs-driven: understanding the hybrid opportunity in blockchain through a case study of Insolar', IEEE Transactions on Engineering Management, DOI: 10.1109/TEM.2020.3003565.

Kwon, J. and Buchman, E. (2018) A Network of Distributed Ledgers, pp.1-41 [online] https://github.com/cosmos/cosmos/blob/master/WHITEPAPER.md (accessed 2 May 2020).

Lone, A.H. and Mir, R.N. (2019) 'Consensus protocols as a model of trust in blockchains', International Journal of Blockchains and Cryptocurrencies, Vol. 1, No. 1, pp.7-21.

Maizels, N. (2018) Secure Multiparty Computation and Shamir's Secret Sharing on Wanchain [online] https://medium.com/wanchain-foundation/secure-multiparty-computationand-shamirs-secret-sharing-on-wanchain-e502012b80ef (accessed 10 March 2020).

McConlogue, P. (2017) Building a Blockchain Singularity with Proof of Distance [online] https://blog.blockcollider.org/building-a-blockchain-singularity-with-proof-of-edit-distance1d60c328de7a (accessed 30 March 2020).

Monika and Bhatia, R. (2020) 'Interoperability solutions for blockchain', International Conference on Smart Technologies in Computing, Electrical and Electronics (ICSTCEE), pp.381-385, DOI: 10.1109/ICSTCEE49637.2020.9277054.

Monrat, A.A., Schelén, O. and Andersson, K. (2019) 'A survey of blockchain from the perspectives of applications, challenges, and opportunities', IEEE Access, Vol. 7, pp.117134-117151, DOI: 10.1109/ACCESS.2019.2936094.

Nakamoto, S. (2008) Bitcoin: A Peer-to-peer Electronic Cash System [online] https://bitcoin.org/ bitcoin.pdf (accessed 14 June 2020).

Olson, K., Bowman, M., Mitchell, J., Amundson, S., Middleton, D. and Montgomery, C. (2018). Sawtooth: An Introduction, The Linux Foundation [online] https:/www.hyperledger.org/wpcontent/uploads/2018/01/Hyperledger_Sawtooth_WhitePaper.pdf (accessed 25 July 2020).

Polkadot (2018) Hybrid PBFT/Aurand [online] https://tokens-economy.gitbook.io/consensus/chainbased-proof-of-stake/hybrid-pbft-aurand (accessed 15 March 2020).

Reecer, D. (2018) An Overview of the Wanchain Cross-chain Implementation Model [online] $\mathrm{https}$ ://medium.com/wanchain-foundation/an-overview-of-the-wanchain-2-0-cross-chainimplementation-model-c455cfd25664 (accessed 12 March 2020). 
Schwartz, D., Youngs, N. and Britto, A. (2014) The Ripple Protocol Consensus Algorithm, Ripple Labs Inc. White Paper, Vol. 5, No. 8, p.151 [online] https://ripple.com/files/ripple_consensus whitepaper.pdf (accessed 26 July 2020).

Spoke, M. and NE Team (2017) Aion: Enabling the Decentralized Internet, White Paper, AION [online] https://whitepaper.io/document/31/aion-whitepaper (accessed 20 March 2020).

Thomas, S. and Schwartz, E. (2015) A Protocol for Interledger Payments [online] https://interledger.org/interledger.pdf (accessed 2 May 2020).

Wanchain, Building Super Financial Markets for the New Digital Economy, Whitepaper version 0.9.0 [online] https://wanchain.org/files/Wanchain-Whitepaper-EN-version.pdf (accessed 8 February 2020).

Weisman, C. (2018) Multichain Functions and Ecosystem [online] https://blog.blockcollider. org/bcgs-8-multichain-functions-and-ecosystem-12-31-18-5647e78469f (accessed 24 March 2020).

What is Cosmos? [online] https://cosmos.network/intro (accessed 27 February 2020).

Wood, G. (2014) Ethereum: A Secure Decentralized Generalised Transaction Ledger, Ethereum project yellow paper, Vol. 151, pp.1-32.

Wood, G. (2016) Polkadot: Vision for a Heterogeneous Multi-chain Framework, White Paper [online] https://github.com/w3f/polkadot-white-paper/blob/master/PolkaDotPaper.pdf (accessed 12 March 2020).

Wu, Y., Zha, Y. and Sun, Y. (2019) A Unifying Hybrid Consensus Protocol, arXiv preprint arXiv: 1906.03251 . 